\title{
Am Anfang war das Leben?
}

\section{Bruno Kesseli}

Dr. med. et lic. phil., Chefredaktor
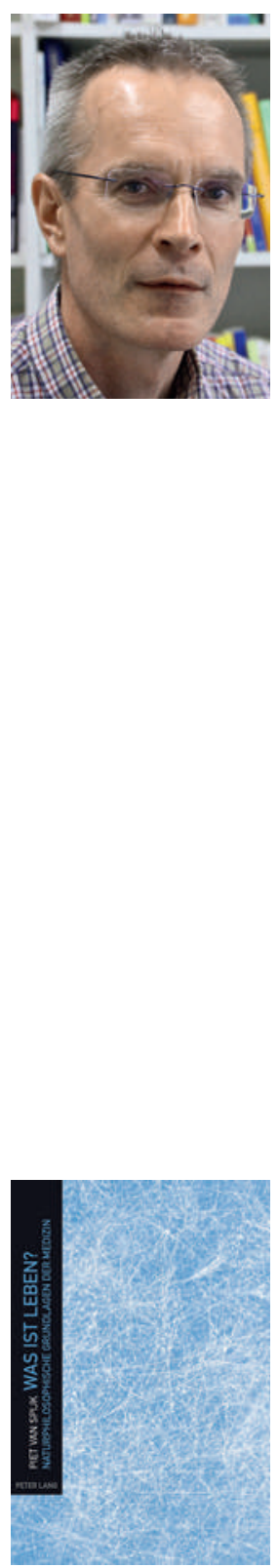

Piet van Spijk Was ist Leben?

Naturphilosophische Grundlagen der Medizin

Bern: Peter Lang; 2014. 297 Seiten. 87 CHF. ISBN 978-3-0343-1321-6

bkesseli[at]emh.ch
Unter den nachhaltigen Erinnerungen an mein Medizinstudium nimmt der erste Auftritt von Professor Jeremias Kägi vor uns frischgebackenen Zweitjahresstudenten einen Spitzenrang ein. Der damalige Direktor des Biochemischen Instituts der Universität Zürich eröffnete seine Vorlesung ungefähr mit folgenden Worten: «Die Biochemie beschäftigt sich mit Vorgängen des Lebens. Was Leben ist, weiss bekanntlich niemand.»

Der ungewöhnliche Einstieg hat dem Ansehen des Professors bei den Studenten sicher nicht geschadet - ganz im Gegenteil. Mir haben sich die Sätze auch deshalb eingeprägt, weil ähnlich unverblümte Eingeständnisse akademischer Grössen in Bezug auf die Grenzen wissenschaftlicher Erkenntnisse Seltenheitswert haben. "Das ist noch nicht im Detail verstanden", lautet der Standardsatz, der in Varianten immer wieder auftaucht. Zuverlässig wie ein alter Kumpel, der einem auf die Schulter klopft und beruhigend ins Ohr flüstert: «Keine Bange, das haben wir gleich.»

Die grossartigen Fortschritte der Biowissenschaften und der Biotechnologie in der jüngeren Vergangenheit, von denen wir alle täglich profitieren, sollen hier keineswegs relativiert werden. Doch während wir im Detail mittlerweile ein fast unheimliches Wissen über das Lebendige angehäuft haben, das Leben in vielen seiner Formen analysieren, beschreiben und in unserem Sinn beeinflussen, gestalten, und instrumentalisieren können, sind grosse Fragen nach wie vor offen. Woher kommt das Leben überhaupt? Wie ist es entstanden? Ist es möglich, aus toter Materie etwas Lebendiges herzustellen? Von umfassendem Verständnis kann wohl erst dann die Rede sein, wenn Fragen dieser Art sicher beantwortet werden können. Ob das je der Fall sein wird, ist ungewiss. Unsere tägliche Erfahrung sagt uns, dass Leben nur aus Leben entstehen kann und alles Lebendige irgendwann stirbt. Das Umgekehrte, dass also etwas Totes plötzlich lebendig wird, hat in meinem Bekanntenkreis noch niemand beobachtet. Die Naturwissenschaft meint aber, davon ausgehen zu müssen, dass genau dies passiert, wenn die Bedingungen stimmen. Täte sie das nicht, müsste sie allenfalls Unbekannte und Kräfte in ihre Gleichungen einführen, die dort wahrlich nichts verloren haben, sondern sich verschiedentlich in wissenschaftlich gesehen abenteuerlichen, religiösen Schriften finden. Wie zum Beispiel in der Bibel, die - Ostern steht vor der Tür die Auferstehung Jesu von den Toten oder die Wiedererweckung des bereits faulenden und deshalb unappetitlich riechenden Lazarus schildert. Da ist man mit Gevatter Zufall, der in der Ursuppe selbstorganisierende Gebilde aus Lipid-Bilayern, Amino- und Nukleinsäuren mit einem Fingerschnippen zum Leben erweckt, doch auf sichererem Terrain. Nicht wahr?

Piet van Spijk hat mit seinem Buch gewissermassen einen Stein ins Wasser geworfen. Es wird interessant sein, zu beobachten, ob die Wellen etwas auslösen.

Neben Naturwissenschaften und Religion gibt es auch noch die Philosophie, die Gewichtiges zum «Problem Leben» zu sagen hat. Und es gibt Philosophen, die auch Ärzte sind (oder umgekehrt). Eine denkbar günstige Voraussetzung für substantielle Beiträge zum Thema, beschäftigen sich Ärztinnen und Ärzte doch täglich intensiv mit dem komplexesten aller Lebewesen. Piet van Spijk, in Luzern tätiger Internist und Philosoph, wird diesem Anspruch gerecht. In seinem 2014 erschienenen Buch Was ist Leben? Naturphilosophische Grundlagen der Medizin setzt er sich umfassend mit den angetönten Fragen und vielen weiteren Aspekten des Phänomens Leben auseinander. Sein Buch ist anspruchsvoll, keine Wochenend-Schnellbleiche für Cüpli-Philosophen. Es ist speziell auch für Ärztinnen und Ärzte lesenswert, werden doch neben theoretischen auch durchaus praktische und medizinrelevante Konsequenzen einer Weltsicht diskutiert, die zwar nicht grundlegend neu ist, aber so etwas wie ein Schattendasein fristet. Das Leben ist das primäre Prinzip, nicht der Tod, lautet die zentrale Hypothese, die für einige Probleme, insbesondere im Zusammenhang mit der Entstehung des Lebens, eine elegante Lösung bietet. Nicht dass das Kägische Diktum damit überholt und die Diskussion darüber, was das Leben denn nun sei, abgeschlossen wäre - das dürfte noch ein klein wenig dauern. Aber Piet van Spijk hat mit seinem Buch gewissermassen einen Stein ins Wasser geworfen. Es wird interessant sein, zu beobachten, ob die Wellen etwas auslösen oder im Meer des Zeitgeistes folgenlos verebben. 